\title{
DOUBLE TAG EVENTS IN TWO-PHOTON COLLISIONS AT LEP
}

\author{
M. WADHWA \\ University of Basel, Klingelbergstrasse 82, \\ CH-4056 Basel, Switzerland \\ E-mail: Maneesh.Wadhwa@cern.ch
}

\begin{abstract}
Double tag events in two photon collisions are studied using the L3 detector at the LEP center of mass energies $\sqrt{s} \simeq 189-202 \mathrm{GeV}$. The cross-section of $\gamma^{*} \gamma^{*}$ collisions is measured at an average photon virtuality $\left\langle Q^{2}\right\rangle=15 \mathrm{GeV}^{2}$. The results are in agreement with Monte Carlo predictions based on perturbative QCD, while the Quark Parton Model alone is insufficient to describe the data. The measurements are compared to the LO and the NLO BFKL calculations.
\end{abstract}

\section{Introduction}

In this paper we present new results on double-tag two-photon events $\mathrm{e}^{+} \mathrm{e}^{-} \rightarrow$ $\mathrm{e}^{+} \mathrm{e}^{-}$hadrons. The data, collected at centreof-mass energies $\sqrt{s} \simeq 189-202 \mathrm{GeV}$, correspond to an integrated luminosity of $401 \mathrm{pb}^{-1}$. Both scattered electrons a are detected in the small angle electromagnetic calorimeters. The virtuality of the two photons, $Q_{1}^{2}$ and $Q_{2}^{2}$, is in the range of $4 \mathrm{GeV}^{2}<$ $Q_{1,2}^{2}<40 \mathrm{GeV}^{2}$.

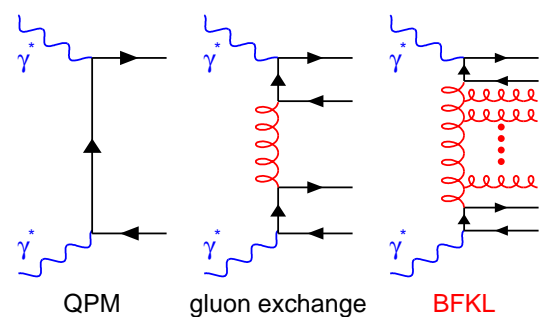

Figure 1. Diagrams for the a) QPM, b) one-gluon exchange and c) BFKL Pomeron processes in a $\gamma^{*} \gamma^{*}$ interaction.

The centre-of-mass energy of the two virtual photons, $\sqrt{\hat{s}}=W_{\gamma \gamma}$, ranges from $5 \mathrm{GeV}$ to $90 \mathrm{GeV}$. The cross-section measurement of the two virtual photons is considered as "golden" process to test the BFKL dynamics 2 . For this scheme the $\gamma^{*} \gamma^{*}$ interaction can be seen as the interaction of two $q \bar{q}$ pairs scattering off each other via multiple gluon

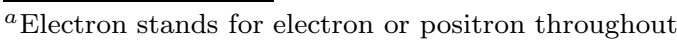
this paper. exchange. (Fig. 11c). In the leading order approximation (LO), the cross-section in the saddle point approximation for the collision of two virtual photons is $\mathrm{B}_{\mathrm{H}}$ :

$$
\sigma_{\gamma^{*} \gamma^{*}}=\frac{\sigma_{0}}{Q_{1} Q_{2} Y}\left(\frac{s}{s_{0}}\right)^{\alpha_{P}-1}
$$

Here

$$
\begin{aligned}
\sigma_{0} & =\text { const } \\
s_{0} & =\frac{K Q_{1} Q_{2}}{y_{1} y_{2}}, \quad Y=\ln \left(s / s_{0}\right) \\
y_{i} & =1-\left(E_{i} / E_{b}\right) \cos ^{2}\left(\theta_{i} / 2\right)
\end{aligned}
$$

where $E_{b}$ is the beam energy, $E_{i}$ and $\theta_{i}$ are the energy and polar angle of the scattered electrons and $\alpha_{P}$ is the "hard Pomeron" intercept; $\mathrm{K}$ is a scale factor which accounts for uncertainity in the BFKL energy scale $s_{0}$. The centre-of-mass energy of the two-photon system is related to the $\mathrm{e}^{+} \mathrm{e}^{-}$centre-of-mass energy $s$ by $\hat{s}=W_{\gamma \gamma}^{2} \approx s y_{1} y_{2}$. In leading order $\left(\alpha_{P}-1\right)=(4 \ln 2) N_{c} \alpha_{s} / \pi$, where $N_{c}$ is the number of colours. Using $N_{c}=3$ and $\alpha_{s}=0.2,\left(\alpha_{P}-1\right) \simeq 0.53$. The born crosssection of one gluon exchange (see Fig. 1 $\mathrm{b}$ ) is independent of $W_{\gamma \gamma}$. Recently, effort has been devoted to improve the exact leading order calculation $\mathrm{l}$ by studying the effect of charm mass and the contribution of longitudinal photon polarization states 6 . Still these effects are not sufficient to describe our previous measurement $\mathrm{B}$. One needs next to leading order corrections(NLO). It turns out that the NLO corrections $\mathrm{G}$ to the intercept 
$" \alpha_{P}-1 "$ are negative for $\alpha_{c}>0.16$. Different techniques 8 , $2,10,11,12,13,14$ have been proposed to improve the NLO calculations in a suitable renormalization scheme thus giving values of $\left(\alpha_{P}-1\right)$ in the range $0.17-0.33$.

\section{Double-tag cross-section}

After selection cuts described in ref 17, we have selected 336 candidate events. The estimated background is 56 events, mainly due to $\mathrm{e}^{+} \mathrm{e}^{-} \rightarrow \mathrm{e}^{+} \mathrm{e}^{-} \tau^{+} \tau^{-}$and misidentified singletag events. The contamination from annihilation processes and lepton channels in two photon collisions is negligible. The preliminary cross-section is measured in the kinematic region limited by:

- $E_{1,2}>30 \mathrm{GeV}, 30 \mathrm{mrad}<\theta_{\text {tag }}<$ $66 \mathrm{mrad}$ and $2 \leq Y \leq 7$

The data is then corrected for efficiency and acceptance with two Monte-Carlo models; PHOJET 15 and Vermaseren(QPM) 16 respectively. The differential cross-sections $\mathrm{d} \sigma\left(\mathrm{e}^{+} \mathrm{e}^{-} \rightarrow \mathrm{e}^{+} \mathrm{e}^{-}+\right.$hadrons $) / \mathrm{d} Y$ are measured in four $\Delta Y$ intervals. As one can be seen in Table 1 and in Fig. 2, none of the models are sufficient to describe the data. The value of the cross-section at $5<Y<7$ exceeds the Monte Carlo prediction by about 3.5 standard deviations.

Table 1. The differential cross-section, $\mathrm{d} \sigma\left(\mathrm{e}^{+} \mathrm{e}^{-} \rightarrow\right.$ $\mathrm{e}^{+} \mathrm{e}^{-}+$hadrons $) / \mathrm{d} Y$ in picobarn measured in the kinematic region defined in the text, at $\sqrt{s} \simeq 189-$ $202 \mathrm{GeV}$. The predictions of the PHOJET and the QPM Monte Carlo models are also listed. The first error is statistical and the second is systematic.

\begin{tabular}{|c|c|c|c|}
\hline$\Delta Y$ & $\begin{array}{c}\text { DATA } \\
\mathrm{d} \sigma / \mathrm{d} Y\end{array}$ & $\begin{array}{c}\text { PHOJET } \\
\mathrm{d} \sigma / \mathrm{d} Y\end{array}$ & $\begin{array}{c}\text { QPM } \\
\mathrm{d} \sigma / \mathrm{d} Y\end{array}$ \\
\hline $2.0-2.5$ & $0.50 \pm 0.07 \pm 0.03$ & 0.40 & 0.32 \\
\hline $2.5-3.5$ & $0.30 \pm 0.03 \pm 0.02$ & 0.29 & 0.17 \\
\hline $3.5-5.0$ & $0.15 \pm 0.02 \pm 0.01$ & 0.14 & 0.05 \\
\hline $5.0-7.0$ & $0.08 \pm 0.02 \pm 0.01$ & 0.03 & 0.006 \\
\hline
\end{tabular}

From the measurement of the $\mathrm{e}^{+} \mathrm{e}^{-} \rightarrow$ $\mathrm{e}^{+} \mathrm{e}^{-}+$hadrons cross-section, $\sigma_{\text {ee }}$, we extract

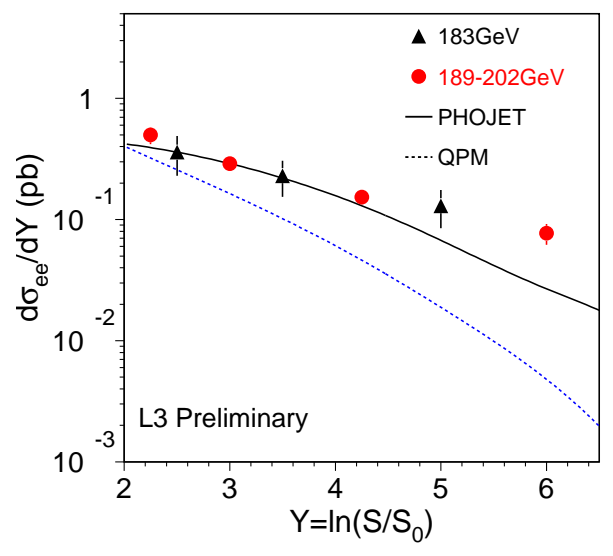

Figure 2. The cross-section of $\mathrm{e}^{+} \mathrm{e}^{-} \rightarrow$ $\mathrm{e}^{+} \mathrm{e}^{-}$hadrons as a function of $Y$ in the kinematical region defined in the text at $\sqrt{s} \simeq 189-202 \mathrm{GeV}$ compared to our previous results $\sqrt{s} \simeq 183 \mathrm{GeV}$. In the figure the predictions of PHOJET (continuos line) and of the QPM (dashed line) are indicated.

the two-photon cross-section, $\sigma_{\gamma^{*}} \gamma^{*}$, by using only the transverse photon luminosity function, $\sigma_{\mathrm{ee}}=L_{T T} \cdot \sigma_{\gamma^{*} \gamma^{*}}$. In Fig. 3 we show $\sigma_{\gamma^{*} \gamma^{*}}$, after subtraction of the QPM contribution as a function of $Y$. Using an average value of $Q^{2},\left\langle Q^{2}\right\rangle=15 \mathrm{GeV}^{2}$ at $\sqrt{s} \simeq$ 189-202 GeV, we calculate the one-gluon exchange contribution with the asymptotic formula. The expectations are below the data. The leading order expectations of the BFKL model, shown as a dotted line in Fig. 3, are too high. By leaving $\alpha_{P}$ as a free parameter and $K=1$, a fit to the data, taking into account the statistical, yields:

$$
\begin{gathered}
\alpha_{P}-1=0.36 \pm 0.02, \quad \chi^{2} / \text { d.o. } f=0.98 / 3 \\
\text { with } \chi^{2} / \text { d.o.f }=0.98 / 3 \text { and if the energy }
\end{gathered}
$$
scale factor $\mathrm{K}$ is a free parameter and $\left(\alpha_{P}-\right.$ $1)=0.53$, a fit to data yields:

$$
K=6.4 \pm 1.0, \quad \chi^{2} / \text { d.o.f }=1.34 / 3
$$

These results are shown in Fig. 3 as a soild and dashed lines respectively. The value of $\left(\alpha_{P}-1\right)$, smaller than expected from the LO BFKL calculation at the saddle point approximation, and the scale factor $K$ much 


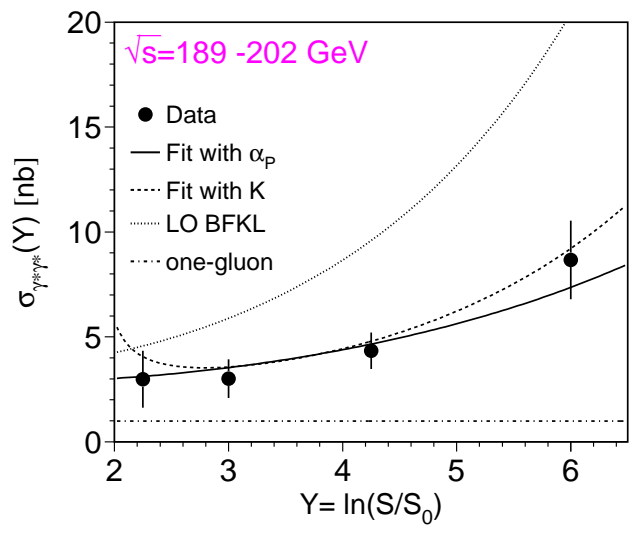

Figure 3. Two-photon cross-sections, $\sigma_{\gamma^{*} \gamma^{*}}$, after the subtraction of the QPM contribution at $\sqrt{s} \simeq$ $189-202 \mathrm{GeV}\left(\left\langle Q^{2}\right\rangle=15 \mathrm{GeV}^{2}\right)$. The data are compared to the predictions of the LO BFKL calculation at saddle point approximation(eq.1)(dotted line) with $\mathrm{K}=1$ and $\left(\alpha_{P}-1\right)=0.53$ and the solid line is the fit to the data of the LO BFKL (eq.1) with $\mathrm{K}=1$ and the coefficient $\left(\alpha_{P}-1\right)$ as a free parameter. The dashed line is the fit with $\left(\alpha_{P}-1\right)=0.53$ and the scale factor $\mathrm{K}$ as a free parameter.

larger than unity indicate that higher order corrections are important. NLO calculations are in progress $14,1.23$ which agree better with the experimental results.

\section{Acknowledgements}

I would like to thank C. H. Lin of his collaboration. This work is supported by the Swiss National Science Foundation.

\section{References}

1. PLUTO Coll., C. Berger et al., Phys. Lett. B 142 (1984) 119; TPC/2 $\gamma$ Coll., D. Bintinger et al., Phys. Rev. Lett. 54 (1985) 763; MD-1 Coll., S.E. Baru et al., Z. Phys. C 53 (1992) 219; TOPAZ Coll., R. Enomoto et al., Phys. Lett. B 368 (1996) 299

2. E.A. Kuraev, L.N. Lipatov and V.S. Fadin, Sov. Phys. JETP 45 (1977) 199; Ya.Ya. Balitski and L.N. Lipatov, Sov. J. Nucl. Phys. 28 (1978) 822
3. S.J. Brodsky, F. Hautmann and D.E. Soper, Phys. Rev. D 56 (1997) 6957

4. J. Bartels, A. De Roeck and H. Lotter, Phys. Lett. B 389 (1996) 742; J. Bartels, A. De Roeck, C. Ewerz and H. Lotter, hep-ph/9710500

5. J. Bartels, C. Ewerz, R. Staritzbichler, hep-ph/0004029

6. L3 Collab., M. Acciarri et al., Phys. Lett. B 453 (1999) 333

7. V. S. Fadin and L. N. Lipatov, Phys. Lett. B 429 (1998) 127; G. Camici and M. Ciafaloni, Phys. Lett. B 430 (1998) 349

8. V. S. Fadin and L. N. Lipatov, Proc. Theory Institute on Deep Inelastic Diffraction, ANL, Argonne, September 14 - 16, 1998; C. R. Schmidt, Phys. Rev. D 60 (1999) 074003; J. R. Forshaw, D. A. Ross and A. Sabio Vera, Phys. Lett. B 455 (1999) 273; S.J. Brodsky et al., JETP Lett. 70 (1999) 15, hep-ph/99101229

9. G. Salam, JHEP 9807 (1998) 019

10. M. Ciafaloni et al., Phys. Rev. D 60 (1999) 114036; M. Ciafaloni and D. Colferai, Phys. Lett. B 452 (1999) 372

11. R. S. Thorne, Phys. Rev. D 60 (1999) 054031

12. G. Altarelli, R. D. Ball and S. Forte, hep$\mathrm{ph} / 0001157$

13. V. T. Kim, L. N. Lipatov and G. B. Pivovarov, hep-ph/9911228 and hep-ph/9911242; V. Kim, private communication

14. N.N. Nikolaev,J. Speth and V.R. Zoller, hep-ph/0001120

15. PHOJET version $1.05 \mathrm{c}$ is used, R. Engel, Z. Phys. C 66 (1995) 203; R. Engel and J. Ranft, Phys. Rev. D 54 (1996) 4244

16. J.A.M. Vermaseren, Nucl. Phys. B 229 (1983) 347

17. Chih-Hsun Lin and M.Wadhwa, L3 Preprint 2568, June 2000 